\title{
Single-pot derivatisation strategy for enhanced gliotoxin detection by HPLC and MALDI-ToF mass spectrometry
}

\author{
Carol Davis • Natasha Gordon • Sinéad Murphy • \\ Ishwar Singh • Kevin Kavanagh • Stephen Carberry • \\ Sean Doyle
}

Received: 27 June 2011 /Revised: 12 August 2011 / Accepted: 16 August 2011 /Published online: 31 August 2011

(C) Springer-Verlag 2011

\begin{abstract}
Gliotoxin is produced by non-ribosomal peptide synthesis and secreted from certain fungi, including Aspergillus fumigatus. It is an epipolythiodioxopiperazine that contains an intact disulphide bridge and is the focus of intense research as a consequence of its negative immunomodulatory properties. Gliotoxin detection is generally enabled by reversed-phase-high-performance liquid chromatography (RP-HPLC), with absorbance detection (220$280 \mathrm{~nm}$ ), or liquid chromatography-mass spectrometry, yet detection is not readily achievable by matrix-assisted laser desorption ionisation-time-of-flight mass spectrometry (MALDI-ToF MS). We have developed a single-pot derivatisation strategy which uses sodium borohydride-mediated reduction of gliotoxin followed by immediate alkylation of exposed thiols by 5 -iodoacetamidofluorescein to yield a stable product, diacetamidofluorescein-gliotoxin (GT-(AF) $)_{2}$ ), of molecular mass $1103.931 \mathrm{Da}((\mathrm{M}+\mathrm{H})+)$. This product is readily detectable by RP-HPLC and exhibits a 6.8-fold
\end{abstract}

Electronic supplementary material The online version of this article (doi:10.1007/s00216-011-5344-1) contains supplementary material, which is available to authorized users.

C. Davis $\cdot$ N. Gordon $\cdot$ S. Murphy $\cdot$ K. Kavanagh $\cdot$ S. Carberry $\cdot$

S. Doyle

Department of Biology, National University of Ireland Maynooth,

Maynooth, Co. Kildare, Ireland

I. Singh

Department of Chemistry,

National University of Ireland Maynooth,

Maynooth, Co. Kildare, Ireland

S. Doyle $(\bowtie)$

National Institute for Cellular Biotechnology, Department

of Biology, National University of Ireland Maynooth,

Maynooth, Co. Kildare, Ireland

e-mail: sean.doyle@nuim.ie

URL: http://biology.nuim.ie increase in molar absorptivity compared with gliotoxin, which results in a higher sensitivity of detection (40 ng; $125 \mathrm{pmoL})$. GT-(AF) $)_{2}$ also fluoresces (excitation/emission, 492:518 nm). Unlike free gliotoxin, the product ( $>800 \mathrm{fmol}$ ) is detectable by MALDI-ToF MS. Sporidesmin A can also be detected by RP-HPLC and MALDI-ToF MS (>530 fmol) using this strategy. We also demonstrate that the strategy facilitates detection of gliotoxin (mean \pm $\mathrm{SD}=3.55 \pm 0.07 \mu \mathrm{g} 100 \mu \mathrm{L}^{-1} ; n=2$ ) produced by $A$. fumigatus, without the requirement for organic extraction of culture supernatants and associated solvent removal. GT-(AF) $)_{2}$ is also detectable $(150 \mathrm{ng} ; 460 \mathrm{pmol})$ by thinlayer chromatography.

Keywords Aspergillus fumigatus .

Epipolythiodioxopiperazine · Gliotoxin · NRPS · Redox ·

Sporidesmin A.

\section{Introduction}

Gliotoxin is secreted by Aspergillus fumigatus and other fungi when grown in culture or during infection of a host organism, where is can exert immunosuppressive effects (Fig. 1) [1, 2]. It is an epipolythiodioxopiperazine (ETP)type toxin (326 Da) which contains a disulphide bridge of unknown origin. This structural feature of gliotoxin plays a highly significant role in mediating the virulence of $A$. fumigatus [3, 4]. Gliotoxin is not immunogenic, and its toxicity in mammalian cells is generally enabled by direct inactivation of essential protein thiols, inhibition of NADPH oxidase assembly, in addition to promotion of redox cycling which leads to hydrogen peroxide formation [1, 5-7]. Sporidesmin A (Fig. 1) is also an ETP-type metabolite which contains a disulphide bridge and is 
<smiles>CN1C(=O)C2CC3=CC=C[C@H](O)[C@H]3N2C(=O)C1CO</smiles>

Gliotoxin

B<smiles>COc1c(Cl)cc2c(c1OC)N(C)C1(O)N3C(=O)C(C)N(C)C(=O)C3C(O)[C@]21O</smiles>

Sporidesmin A
C
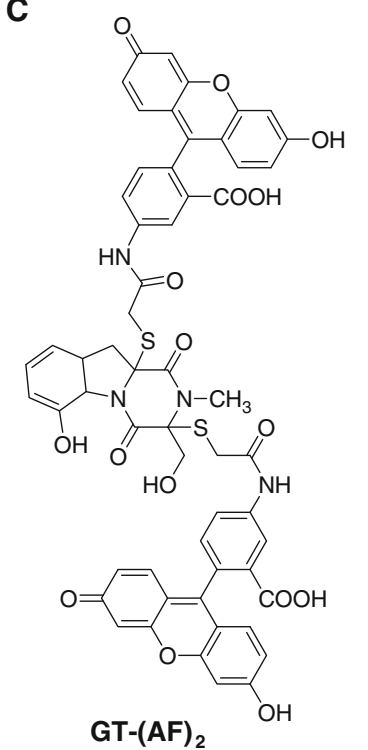

Fig. 1 A Gliotoxin, B sporidesmin A, and $\mathbf{C}$ proposed structure of diacetamidofluorescein-gliotoxin (GT-(AF) $)_{2}$ )

produced by the animal disease-causing fungus, Pithomyces chartarum [3].

The detection of fungal-specific peptide biomarkers such as gliotoxin represents an emerging strategy for the reliable diagnosis of fungal infection which, to date, has proved to be extremely difficult $[8,9]$. Gliotoxin detection is normally by reversed-phase high-performance-liquid chromatography (RP-HPLC) with UV absorbance detection (220-280 nm), gas chromatography-mass spectrometry or liquid chromatography-mass spectrometry (LC-MS) analysis [1013]. More specifically, gliotoxin detection following RPHPLC separation is usually carried out at $254 \mathrm{~nm}$ [10]. The immunological detection of gliotoxin by ELISA has been demonstrated, and here, the limit of detection was $10 \mu \mathrm{g} \mathrm{mL}^{-1}$ [14]. In addition, Lewis et al. showed that gliotoxin was present in sera and lungs of mice suffering from experimentally induced invasive aspergillosis by LCMS analysis [15]. These authors also demonstrated that gliotoxin could be detected, by LC-MS, in cancer patients with either proven or probable invasive aspergillosis [15].

Studies on gliotoxin biosynthesis, which have demonstrated the role of the non-ribosomal peptide synthetase, GliP, in mediating the initial step of gliotoxin formation, have also deployed LC-MS to detect the presence and absence of gliotoxin in wild-type and mutant $(\Delta g l i P)$ cultures from A. fumigatus [16]. Moreover, using a chemical reduction and alkylation strategy, thiol and disulphide absence has been confirmed in a gliotoxin-like metabolite isolated from an A. fumigatus mutant deficient in gliotoxin biosynthesis [17]. To date, matrix-assisted laser desorption ionisation-time-of-flight mass spectrometry (MALDI-ToF MS) has not been used for the detection of gliotoxin, as matrix components can interfere with the detection of low molecular mass metabolites and gliotoxin fragments during ionisation, thereby rendering detection impossible [16, 18].

The constituent disulphide bridge of gliotoxin can be cleaved and converted to two thiol groups by reduction with compounds such as Cleland's reagent/dithiothreitol or $\beta$-mercaptoethanol [19]. However, these low molecular mass molecules cannot be removed by dialysis or gel filtration from reduced gliotoxin, and their presence will interfere with any subsequent chemical modification (e.g., alkylation) of exposed thiols. It has also been demonstrated that glutathione-mediated reduction of gliotoxin is possible but results in the formation of mixed disulphides [20,21] which would be refractory to subsequent modification through blocked thiols. Alternative reducing agents compatible with post-reduction labelling are therefore required, and Woodcock et al. have demonstrated that sodium borohydride $\left(\mathrm{NaBH}_{4}\right)$ can reduce both gliotoxin and sporidesmin A [22].

In the present work, $\mathrm{NaBH}_{4}$ was used to reduce the disulphide bridge of gliotoxin to generate thiol groups which could react with $5^{\prime}$-iodoacetamidofluorescein (5'IAF) to yield a stable derivative. This reaction product is detectable at higher sensitivity than unlabelled gliotoxin, by RP-HPLC, and could also be detected by MALDI-ToF MS and thin-layer chromatography analysis (TLC).

\section{Materials and methods}

Materials and reagents

All reagents were obtained from Sigma-Aldrich, Dorset, UK, unless otherwise indicated. 5'-IAF was prepared in dimethyl sulfoxide (DMSO) at 3-10 mg mL $\mathrm{mL}^{-1} \cdot \mathrm{NaBH}_{4}$ $(500 \mathrm{mM})$ was prepared in HPLC-grade water and used within 30 min of preparation. A. fumigatus strains Af293 (wild-type) and $\Delta g l i Z$ (a kind gift from Professor Nancy Keller, University of Wisconsin-Madison), a binuclear cluster domain transcription factor-deficient mutant incapable of gliotoxin biosynthesis [23], were cultured as described elsewhere [17, 24]. Sporidesmin A was purchased from AgResearch (Hamilton, New Zealand).

Reduction and alkylation of gliotoxin under organic or aqueous conditions

Solutions containing gliotoxin $(100 \mu \mathrm{L} ; 100 \mu \mathrm{g} \mathrm{mL}$ methanol; $30.6 \mathrm{nmoL}$ gliotoxin) were prepared. To these were added $2.5 \mu \mathrm{L} 500 \mathrm{mM} \mathrm{NaBH}{ }_{4}\left(1.2 \mu \mathrm{mol} \mathrm{NaBH} \mathrm{N}_{4}\right)$, followed by gentle mixing by vortexing and incubation for $60 \mathrm{~min}$ at room temperature (occasionally $50 \mathrm{mM} \mathrm{NaBH}_{4}$ 
was used as reductant, as indicated). Labelled sporidesmin A was prepared under identical conditions. No $\mathrm{NaBH}_{4}$ was added when the intact disulphide bridge of gliotoxin was required (i.e. as negative control), and gliotoxin preparations were also independently reduced using $6 \mu \mathrm{L}$ dithiothreitol (DTT; $10 \mathrm{mM}$ in deionised water), which contains free thiol groups, for comparison with $\mathrm{NaBH}_{4}$ as reducing agent. 5'-IAF $\left(20 \mu \mathrm{L}, 3 \mathrm{mg} \mathrm{mL}^{-1} ; 120 \mathrm{nmol}\right)$ was added to reduced or oxidised gliotoxin preparations (30.6 nmol), followed by vortexing of the resultant mixtures briefly and incubation for $40 \mathrm{~min}$ in the dark at room temperature prior to HPLC analysis. Alkylation of reduced gliotoxin was also undertaken as described above using excess iodoacetamide (400 nmol) instead of 5'-IAF. Tris(2-carboxyethyl) phosphine hydrochloride (TCEP.HCl; Thermo Scientific) could also be used as reducing agent (125 nmol per reaction) prior to 5'-IAF-mediated alkylation.

RP-HPLC analysis of culture supernatants (48 h; adjusted to $\mathrm{pH} 7.5$ using 0.1 volume $1 \mathrm{MNa}_{2} \mathrm{HPO}_{4} \mathrm{pH} 7.5$ ) of $A$. fumigatus Af293, spiked with gliotoxin and following $\mathrm{NaBH}_{4}(500 \mathrm{mM})$ reduction and subsequent labelling with 5'-IAF was also undertaken. Here, gliotoxin $\left(327 \mu \mathrm{g} \mathrm{mL}^{-1}\right.$; $50 \mu \mathrm{L}$ ) was added to $50 \mu \mathrm{L}$ A. fumigatus culture supernatants $\mathrm{pH} 7.5$, followed by addition of $50 \mu \mathrm{L}$ dimethyl sulfoxide. Sodium borohydride $(3.3 \mu \mathrm{L} ; 50$ or $500 \mathrm{mM}$ ) was then added followed by $10 \mu \mathrm{L} \mathrm{5'-IAF}\left(10 \mathrm{mg} \mathrm{mL}^{-1}\right)$. Modification of endogenous gliotoxin in culture supernatants, under aqueous conditions, via sodium borohydride reduction and 5'-IAF labelling was undertaken as follows$2.5 \mu \mathrm{L} 500 \mathrm{mM} \mathrm{NaBH}_{4}$ and $20 \mu \mathrm{L} \mathrm{5'-IAF}\left(3 \mathrm{mg} \mathrm{mL}^{-1}\right.$; $120 \mathrm{nmol})$ were added per $100 \mu \mathrm{L}$ test specimen $(A$. fumigatus culture supernatants, $\mathrm{pH}$ 7.5) and incubated sequentially as indicated above. Endogenous gliotoxin was also chloroform-extracted from A. fumigatus culture supernatants and resuspended in methanol [17]. Extensive assay validation and investigation included evaluation of alternative chromatographic conditions to detect labelled gliotoxin, compatibility with MALDI-ToF MS, sensitivity of detection, reproducibility, reductive alkylation of gliotoxin under aqueous conditions, sporidesmin A compatibility and TLC detection.

\section{RP-HPLC analysis}

Reversed-phase HPLC used solvent A $0.1 \%(v / v)$ trifluoroacetic acid in HPLC-grade water and solvent B $0.1 \%(v / v)$ trifluoroacetic acid in $100 \%(v / v)$ acetonitrile. Chromatography, using a $\mathrm{C}_{18}$ column (Agilent Zorbax Eclipse XDBC18; 5- $\mu \mathrm{m}$ particle size; $4.6 \times 15 \mathrm{~mm}$ ) was carried out using an Agilent Series 1,200 HPLC system equipped with diode array (DAD) and fluorescence detectors. Gradient HPLC conditions (three) were as follows: method 1, 35-60\% solvent $\mathrm{B}$ over $6 \mathrm{~min}(\Delta \% \mathrm{~B} / \min =4.0)$; method $2,5-100 \%$ solvent $\mathrm{B}$ over $20 \mathrm{~min}(\Delta \% \mathrm{~B} / \mathrm{min}=4.75)$ or method 3 , over $24 \mathrm{~min}(\Delta \% \mathrm{~B} / \mathrm{min}=4.0)$ and were employed for gliotoxin, reduced gliotoxin, and labelled gliotoxin separation followed by absorbance detection ( 220 or $254 \mathrm{~nm}$ ) plus fluorescence detection (excitation, $492 \mathrm{~nm}$; emission, $518 \mathrm{~nm}$ ). Isocratic conditions were always established prior to specimen injection and the column washed and re-equilibrated prior to subsequent specimen injection. Sporidesmin A was similarly analysed. Specimen injection volume was $20 \mu \mathrm{L}$, unless otherwise noted, at a solvent flow rate of $1 \mathrm{~mL} \mathrm{~min}^{-1}$.

\section{MALDI-ToF MS}

Mass spectrometry was carried out using an Ettan ${ }^{\mathrm{TM}}$ MALDIToF mass spectrometer (Amersham Biosciences (Europe) $\mathrm{GmbH}$, Freiburg, Germany). Specimens $(0.5 \mu \mathrm{L})$, neat or diluted up to $1 / 200$ in $0.1 \%(v / v)$ trifluoroacetic acid, for mass determination were layered upon $0.5 \mu \mathrm{L} \alpha$-cyano-4-hydroxycinnamic acid (5 mg $200 \mu \mathrm{L}^{-1} 50 \%(v / v)$ acetonitrile in $0.1 \%$ $(v / v)$ aqueous trifluoroacetic acid), previously deposited onto mass spectrometry slides and allowed to dry prior to delayed extraction, reflectron $\mathrm{ToF}$ analysis at $20 \mathrm{kV}$ [24]. Internal calibrants, angiotensin III and adrenocorticotropic hormone fragments 18-39, were used to calibrate spectra.

\section{Thin-layer chromatography analysis}

TLC analysis of reduced and labelled gliotoxin, 5'-IAF only, and unreduced gliotoxin in the presence of 5'-IAF was performed. TLC analysis used Merck silica gel 60-F254 TLC plates (aluminium backed). Solvent systems were dichloromethane/methanol (90:10; containing $100 \mu \mathrm{L}$ acetic acid per $10 \mathrm{~mL}$ ) or dichloromethane/methanol (94:6). The sample application volume was $1 \mu \mathrm{L}$. Images were obtained using a Fluorescence Scanner (Typhoon Variable Mode Imager; GE Healthcare) at excitation and emission wavelengths at 488 and $520 \mathrm{~nm}$, respectively (sensitivity setting, $600 \mathrm{~V} ; 50 \mu \mathrm{m}$ pixel size).

\section{Results}

Alkylation of $\mathrm{NaBH}_{4}$-reduced gliotoxin enhances detection

$\mathrm{NaBH}_{4}$-mediated reduction enables the subsequent alkylation of exposed thiols on gliotoxin using 5'-IAF; moreover, removal of the reducing agent is not required prior to alkylation. Figure $2 \mathrm{a}$ illustrates the formation of 5'-IAFlabelled gliotoxin (retention time $\left(R_{\mathrm{T}}\right)=5.18 \mathrm{~min}$ ), following RP-HPLC separation and detection at $254 \mathrm{~nm}$. Unreacted $5^{\prime}$ IAF is also visible upon chromatography $\left(R_{\mathrm{T}}=4.66 \mathrm{~min}\right)$. Labelled gliotoxin is not present when the disulphide bridge of gliotoxin remains intact (in the absence of $\mathrm{NaBH}_{4}$ ) and 

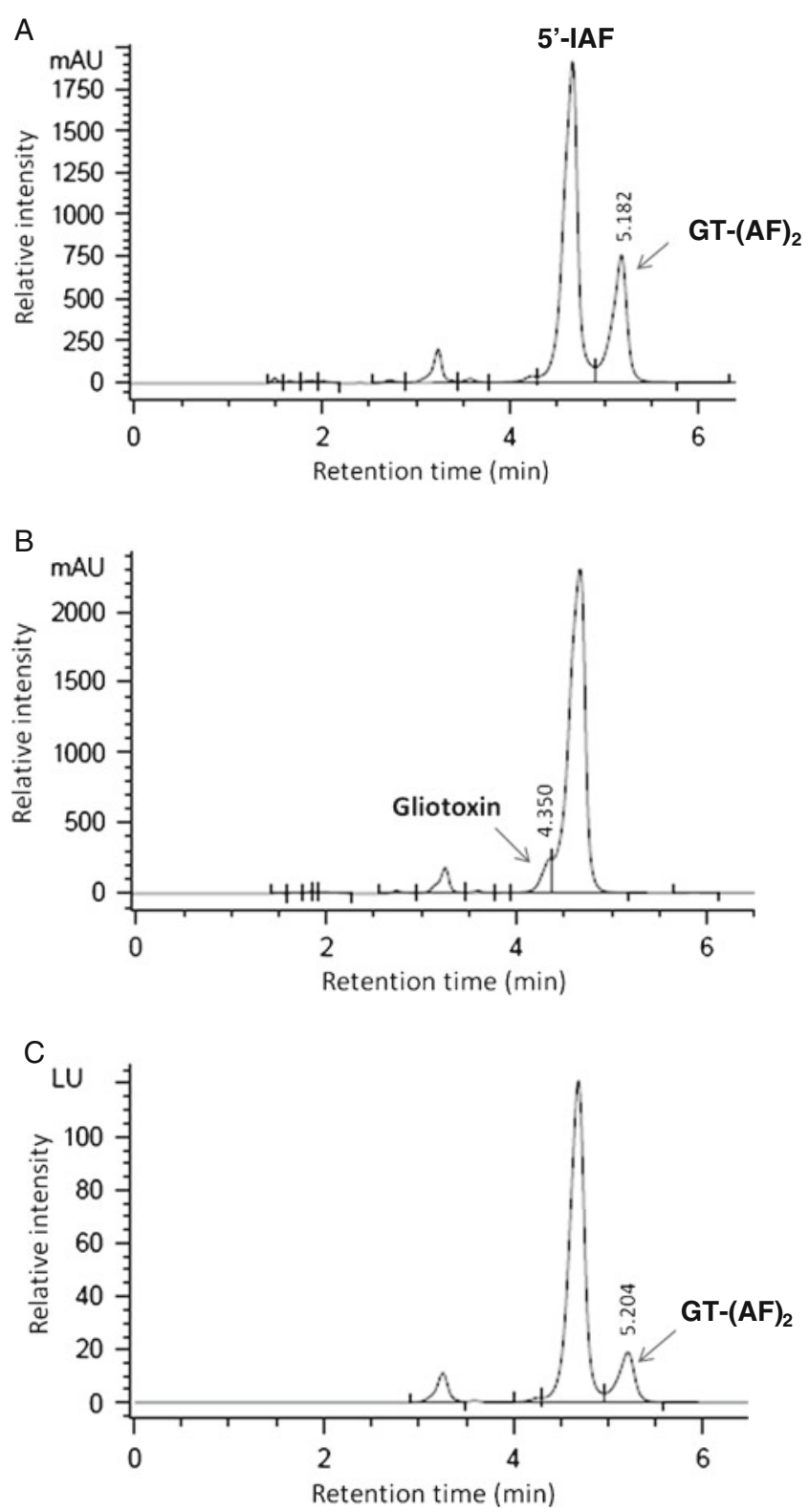

Fig. 2 RP-HPLC analysis of pure gliotoxin (Sigma-Aldrich) with A and without $\mathbf{B}$ sodium borohydride-mediated reduction prior to 5 '-IAF labelling. A Gliotoxin $+\mathrm{NaBH}_{4}+5^{\prime}$-IAF: Gliotoxin disulphide bridge is reduced and $\mathrm{SH}$ groups (thiols) alkylated by $5^{\prime}$-IAF. The fluorescently labelled form of gliotoxin $\left(\mathrm{GT}-(\mathrm{AF})_{2}\right)$ was detectable at $254 \mathrm{~nm}$ with a retention time of $5.18 \mathrm{~min}$ as indicated in (A). B Gliotoxin disulphide intact: No fluorescent labelling of gliotoxin possible. Free gliotoxin (GT) is detectable at $254 \mathrm{~nm}$ with a retention time of $4.35 \mathrm{~min}$. Identical amounts of gliotoxin were present in each assay, yet the labelled form exhibits higher absorbance, compared with free gliotoxin, at $254 \mathrm{~nm}$. C Fluorescence detection of labeled gliotoxin following HPLC separation. Excitation and emission 492/ $518 \mathrm{~nm}$. Fluorescently labelled gliotoxin is evident at retention time of $5.20 \mathrm{~min}$ (slightly later than that for a $254 \mathrm{~nm}$ detection $(5.18 \mathrm{~min})$ as the fluorescence detector was in series after DAD)

therefore unavailable for alkylation (Fig. 2b). However, free gliotoxin is evident in Fig. $2 \mathrm{~b}\left(R_{\mathrm{T}}=4.35 \mathrm{~min}\right)$. Although the amounts $(10 \mu \mathrm{g})$ of gliotoxin initially present in reaction mixtures were identical, absorbance of 5'-IAF labelled gliotoxin (peak area $(\operatorname{mean} \pm \mathrm{SD})=8,772 \pm 606 ; n=3$ ) was enhanced relative to the unlabelled reduced or oxidised form (peak area $($ mean $\pm \mathrm{SD})=1,287 \pm 122 ; n=3)$. This observed increase in $\mathrm{A}_{254 \mathrm{~nm}}$ of labelled gliotoxin is clearly due to an increase in the molar extinction coefficient $(\varepsilon)$ relative to free gliotoxin and indicates a 6.8-fold increase in sensitivity of detection for labelled, compared with free, gliotoxin detection. The labelled form of gliotoxin also fluoresces, although it appears that the quantum yield of the fluorescein component is suppressed at the low $\mathrm{pH}$ conditions employed for RPHPLC (Fig. 2c). Here, fluorescently labelled gliotoxin is detected at $R_{\mathrm{T}}=5.20 \mathrm{~min}$, and no fluorescently labelled gliotoxin is evident without prior reduction (data not shown). Thus, identification of labelled gliotoxin at $254 \mathrm{~nm}$ enhances the specificity, as well as sensitivity, of the method over preexisting strategies for direct gliotoxin detection.

\section{Modified gliotoxin is detectable by MALDI-ToF MS}

Detection of gliotoxin by MALDI-ToF MS is confounded by both small molecular size and ionisation-induced fragmentation, however, alkylation of gliotoxin results in a stable molecular species readily detectable by MALDI-ToF MS. Both thiol groups of reduced gliotoxin were covalently alkylated by $5^{\prime}$-IAF to form a diacetamidofluorescein derivative of gliotoxin, confirmed by the appearance of a species of $m / z 1103.931\left((\mathrm{M}+\mathrm{H})^{+}\right)$which corresponds precisely to the protonated form of doubly acetamidofluorescein-labelled gliotoxin (i.e. GT-(AF) $)_{2}(1102.93 \mathrm{Da})$, as determined by MALDI-ToF MS analysis (Fig. 3). The proposed molecular structure of GT-(AF) $)_{2}$ is shown in Fig. 1c. Reduction and 5'IAF treatment of a gliotoxin-deficient strain (A. fumigatus $\Delta g l i Z$ [23]) did not yield any GT-(AF $)_{2}$, thereby confirming the specificity of gliotoxin detection using this strategy (data not shown). No product was detectable by MALDI-ToF MS either in the absence of prior reduction or the absence of 5'IAF (data not shown). Gliotoxin was also labelled by iodoacetamide-mediated alkylation, resulting in complete disappearance of oxidised gliotoxin from solution; however, no evidence of absorbance enhancement of alkylated gliotoxin was apparent in this case (data not shown). Neither was any molecular species of $\mathrm{m} / \mathrm{z} 384.96$ or 443.02 , which would correspond to the mass of mono- or diacetamido-gliotoxin, respectively, detectable by MALDI-ToF MS. Thus, it appears that only 5'-IAF, and not iodoacetamide, is a suitable agent to stabilise gliotoxin for subsequent MALDI analysis.

Derivatised sporidesmin A detection by RP-HPLC and MALDI-ToF MS

Sporidesmin A underwent $\mathrm{NaBH}_{4}$-mediated reduction and was also subjected to alkylation. RP-HPLC analysis using 
A

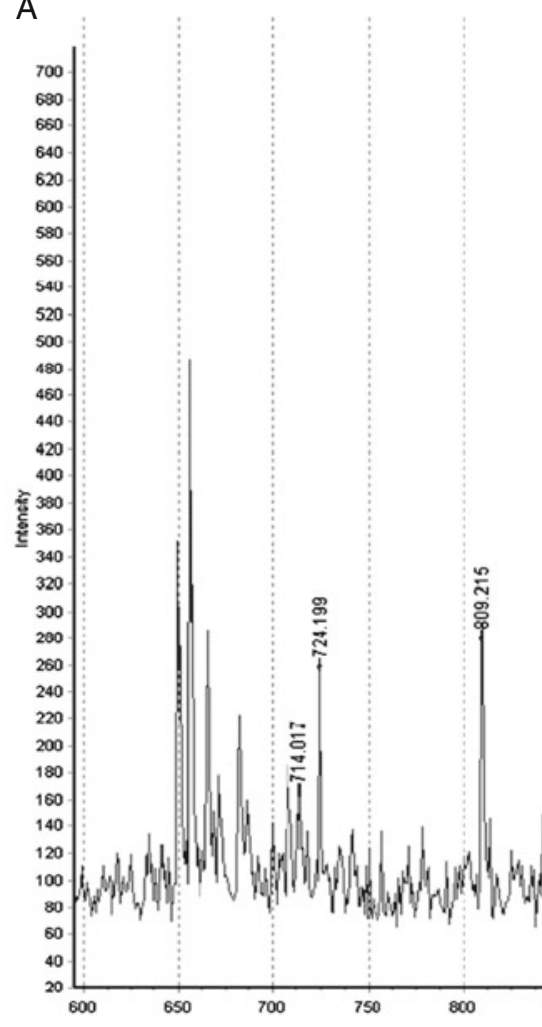

GT-(AF) $)_{2}$

1103.931
Fig. 3 MALDI-ToF mass spectrum of GT-(AF) $)_{2}(800 \mathrm{fmol})$ prepared by reduction and alkylation of gliotoxin. A molecular species of $\mathrm{m} / \mathrm{z}$ $1103.931((\mathrm{M}+\mathrm{H})+)$ is detectable which corresponds precisely to protonated diacetamidofluorescein-gliotoxin. No product was detectable either in the absence of prior reduction or the absence of $5^{\prime}$-IAF method 1 demonstrated that reduction resulted in a shift in retention time from 7.27 to $5.48 \mathrm{~min}$ (Fig. 4a, b) and that sporidesmin A could be derivatised with 5'-IAF (only after reduction) to yield a product, sporidesmin $\mathrm{A}-(\mathrm{AF})_{2}$, which eluted at $7.96 \mathrm{~min}$ (Fig. 4c, d). Sporidesmin A-(AF) (>530 fmol) was detectable by MALDI-ToF MS, as described for GT-(AF) $)_{2}$, as a charged species at $\mathrm{m} / \mathrm{z}$ 1251.145, which corresponds precisely to the predicted protonated mass of the derivative (Fig. 4e). No sporidesmin A-(AF $)_{2}$ was detectable without the presence of $\mathrm{NaBH}_{4}$ in the reaction mixtures (data not shown).

$\mathrm{NaBH}_{4}$ is the preferred reductant for gliotoxin

Data in Fig. S1 (Electronic Supplementary Material) illustrate that GT- $(\mathrm{AF})_{2}$ is detectable at $254 \mathrm{~nm}$ with a retention time of 15.24 min using either DTT (peak area $($ mean $\pm \mathrm{SD})=1,833 \pm 1,543 ; n=3) \quad$ (Fig. S1a, Electronic Supplementary Material) or $\mathrm{NaBH}_{4}$ (peak area (mean \pm $\mathrm{SD})=8,772 \pm 606 ; n=3$ ) (Fig. S1b, Electronic Supplementary Material) as reducing agent. However, it is clear that the use of DTT as reductant results in diminished formation of labelled gliotoxin (fivefold lower), most likely due to a reduced rate of disulphide bridge cleavage relative to $\mathrm{NaBH}_{4}$ and competition with gliotoxin-thiols for reaction with 5'-IAF. Moreover, residual oxidised gliotoxin $\left(R_{\mathrm{T}}=\right.$ $14.42 \mathrm{~min}$ ) was present (Fig. S1a, Electronic Supplementary Material) indicating that DTT is an inferior reductant compared with $\mathrm{NaBH}_{4}$ for cleavage of the disulphide bridge on gliotoxin. $\mathrm{NaBH}_{4}$-mediated reduction of gliotoxin is complete within a maximum of 15 min (mean \pm $\mathrm{SD}=1.5 \pm 0.23 \mu \mathrm{g} ; n=3$ ), and reduced gliotoxin does not exhibit any evidence of re-oxidation for up to $2 \mathrm{~h}$ postreduction (mean $\pm \mathrm{SD}=1.5+0.11 \mu \mathrm{g} ; n=3$ ). TCEP addition also resulted in formation of reduced gliotoxin which was subsequently alkylated with 5'-IAF (data not shown); however, use of TCEP required reaction $\mathrm{pH} 7.6$ when gliotoxin modification was undertaken in organic solvents. No $\mathrm{pH}$ adjustment was necessary using $\mathrm{NaBH}_{4}$ under these conditions.

\section{Assay validation}

A calibration curve for gliotoxin detection using sequential reduction and labelling is shown in Fig. 5, and a linear response is observed between 0 and 
Fig. 4 RP-HPLC analysis of pure sporidesmin A A prior to and $\mathbf{B}$ post-sodium borohydridemediated reduction prior to 5'IAF labelling. C Sporidesmin $\mathrm{A}+\mathrm{NaBH}_{4}+5^{\prime}$-IAF: disulphide bridge is reduced and $\mathrm{SH}$ groups (thiols) alkylated by 5'-IAF. Sporidesmin A-(AF) $)_{2}$ was detectable at $254 \mathrm{~nm}$ with a retention time of $7.96 \mathrm{~min}$. D Sporidesmin A+5'-IAF: only free sporidesmin $\mathrm{A}$ is present (7.3 min). Without reduction, sporidesmin $\mathrm{A}-(\mathrm{AF})_{2}$ is absent. $\mathbf{E}$ MALDI-ToF mass spectrum of sporidesmin $\mathrm{A}-(\mathrm{AF})_{2}$ prepared by reduction and alkylation (530 fmol). A molecular species of $m / z 1251.145((\mathrm{M}+\mathrm{H})+)$ is detectable which corresponds precisely to protonated diacetamidofluorescein-sporidesmin A. No product was detectable either in the absence of prior reduction or the absence of 5 '-IAF (data not shown)
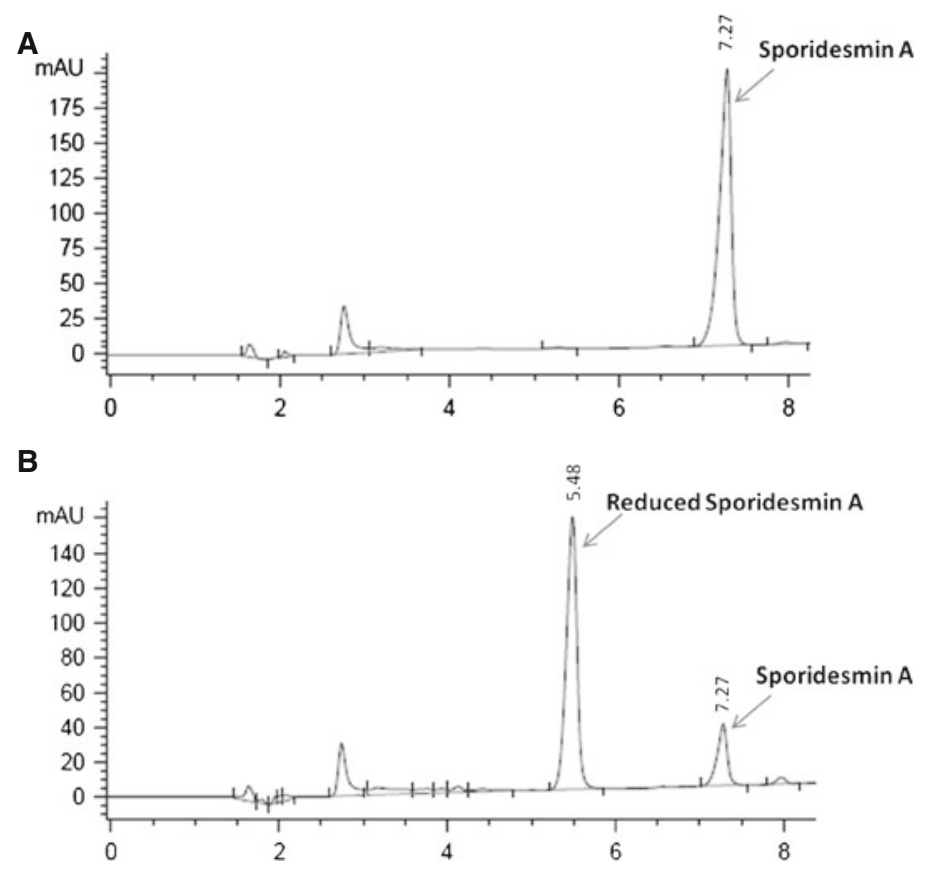

C
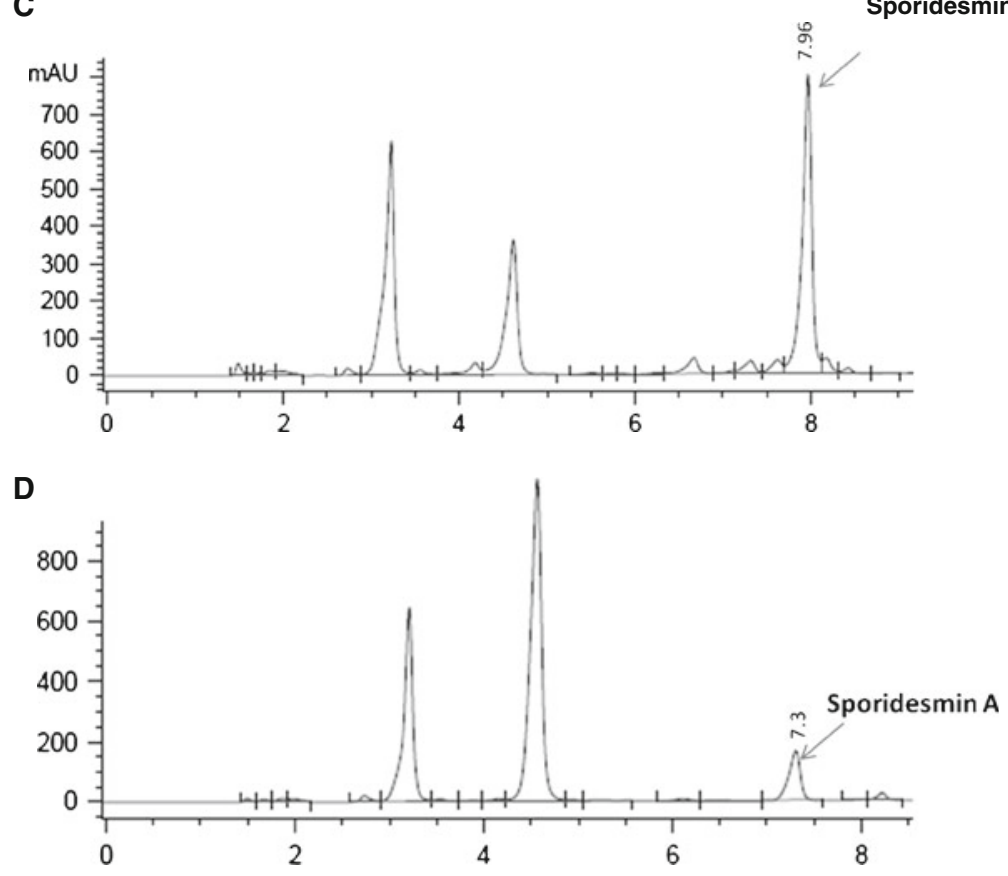

2,000 pmoL gliotoxin, following RP-HPLC at $254 \mathrm{~nm}$. The limit of detection for free gliotoxin is $125 \mathrm{pmoL}$ (40 ng). Intra-assay reproducibility $(n=5)$ was determined to range between $13 \%$ and $14 \% \mathrm{CV}$, and interassay reproducibility $(n=5)$ was calculated to be between $13 \%$ and $16 \% \mathrm{CV}$, between for 10-30 nmol gliotoxin (Table S1). GT-(AF) 2 exhibited a mean post-synthesis stability of $88 \%$ during storage at $20-25{ }^{\circ} \mathrm{C}$ for $24 \mathrm{~h}$ in the dark. Thus, for long-term storage (up to 1 month) of GT$(\mathrm{AF})_{2},-20{ }^{\circ} \mathrm{C}$ is recommended.
Reduction and alkylation facilitates detection of gliotoxin produced by $A$. fumigatus

To further confirm the utility of the method for gliotoxin detection, organic extracts from 48-h cultures of $A$. fumigatus Af293 were prepared and subjected to reduction and alkylation with 5'-IAF (Fig. S2, Electronic Supplementary Material). Although GT-(AF) $)_{2}$ exhibits enhanced absorbance at 220 versus $254 \mathrm{~nm}$, the majority of analyses were performed at the latter wavelength to minimise solvent 


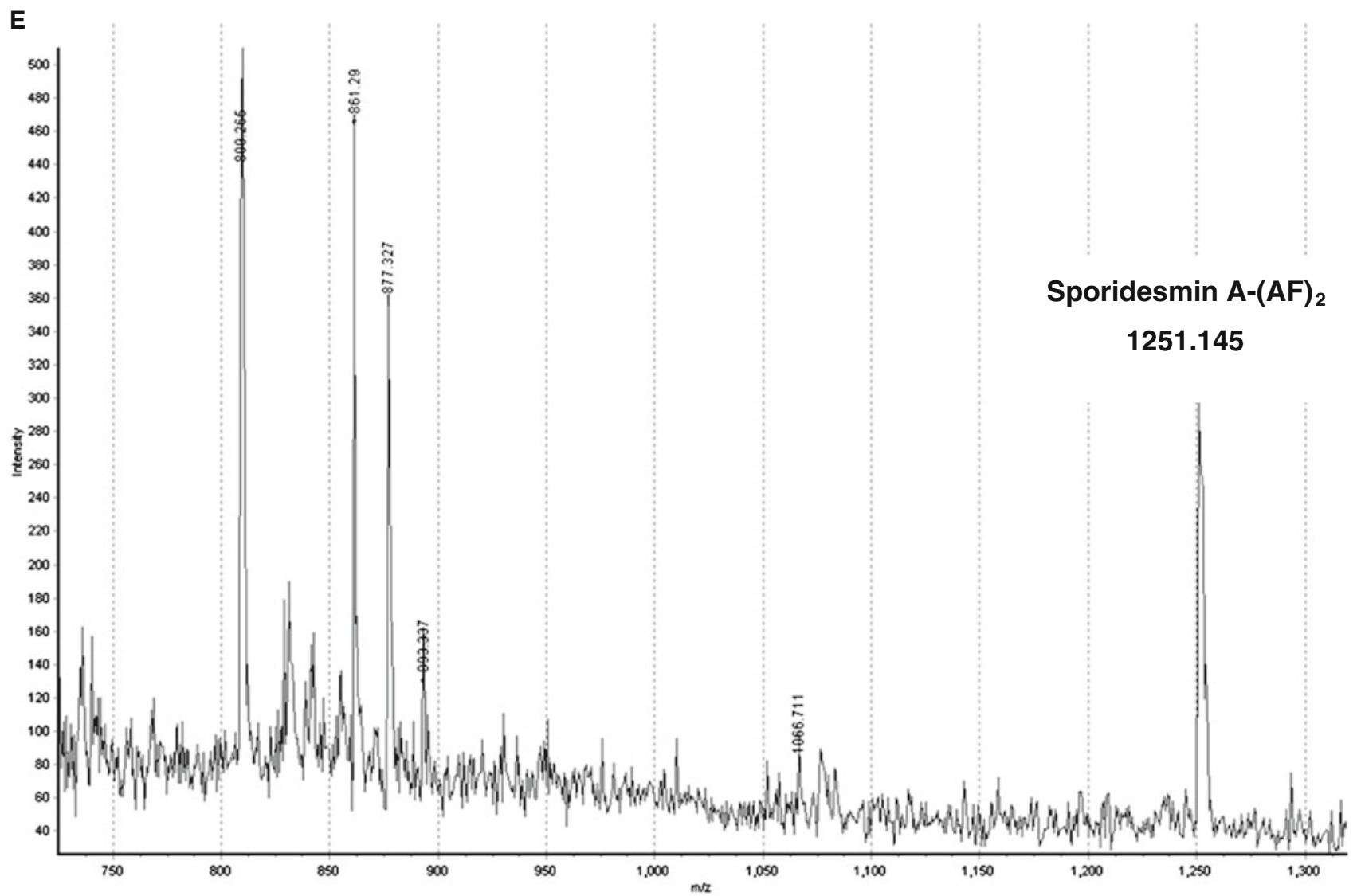

Fig. 4 (continued)

absorbance effects and yield clearer chromatograms. Data presented in Fig. 6a illustrate that spiked gliotoxin can also be detected in A. fumigatus culture supernatants without the need for prior extraction. This strategy required the upward $\mathrm{pH}$ adjustment of culture supernatants (final $\mathrm{pH} 7.5$ ) and addition of DMSO to ensure solubility of 5'-IAF during the labelling. Interestingly, it was noted that use of stock solutions of $\mathrm{NaBH}_{4}(500 \mathrm{mM}$ as opposed to $50 \mathrm{mM})$ specifically

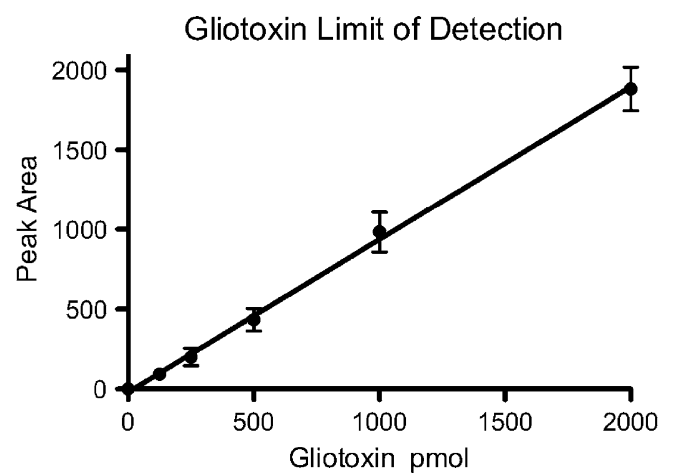

Fig. 5 Calibration curve for gliotoxin detection by sequential reduction and alkylation ( $0-2,000$ pmoL gliotoxin). Assay conditions are described in "Materials and methods", and error bars represent mean amount $\pm \mathrm{SD}(n=3)$ suppressed the absorbance of unreacted 5'-IAF while that of GT-(AF) $)_{2}$ was unaffected (Fig. 6b). Detection and quantitation of native gliotoxin in A. fumigatus culture supernatants (mean $\pm \mathrm{SD}=3.55 \pm 0.07 \mu \mathrm{g} / 100 \mu \mathrm{L} ; n=2$ ), without the need for prior organic extraction, and organic solvent (DMSO) addition to reaction mixtures, was also achieved (Fig. 6c). Addition of lower amounts of 5'-IAF, as a consequence of lower amounts of endogenous gliotoxin, obviated the requirement to add DMSO to maintain $5^{\prime}$-IAF solubility. Labelled gliotoxin was absent without prior reduction (Fig. 6d).

Thin-layer chromatography

Finally, a TLC method for the detection of labelled gliotoxin has also been developed (Fig. S3, Electronic Supplementary Material). Solvent optimisation was necessary in order to achieve the required resolution between GT-(AF) $)_{2}$ and $5^{\prime}$-IAF (data not shown). The optimal method utilised Merck silica gel 60-F254 TLC plates (aluminium backed), a dichloromethane/methanol/acetic acid (90:10:1) solvent system, can be completed in $30 \mathrm{~min}$ and represents a strategy for highthroughput, simultaneous analysis of multiple test specimens. Detection of $150 \mathrm{ng}$ ( $460 \mathrm{pmoL}) \mathrm{GT}-(\mathrm{AF})_{2}$ was evident, by fluorescence scanning (sensitivity setting, $600 \mathrm{~V}$ ), only when 
Fig. 6 Optimised RP-HPLC detection of GT-(AF) $)_{2}$ prepared under aqueous conditions. A RP-HPLC analysis (method 2) of culture supernatant ( $48 \mathrm{~h}$; adjusted to $\mathrm{pH} 7.5$ ) of $A$. fumigatus Af293 strain, spiked with gliotoxin and following $\mathrm{NaBH}_{4}$ $(50 \mathrm{mM})$ reduction and subsequent labelling with 5 '-IAF. The presence of GT-(AF) $)_{2}$ was observed at $15.52 \mathrm{~min}$. B RP-

HPLC analysis of culture supernatants ( $48 \mathrm{~h}$; adjusted to $\mathrm{pH} 7.5$ ) of A. fumigatus Af293, spiked with gliotoxin and following $\mathrm{NaBH}_{4}(500 \mathrm{mM})$ reduction and subsequent labelling with 5'-IAF. Gliotoxin $\left(327 \mu \mathrm{g} \mathrm{mL}^{-1} ; 50 \mu \mathrm{L}\right)$ was added to $50 \mu \mathrm{L}$ A. fumigatus culture supernatant $\mathrm{pH} 7.5$ followed by addition of $50 \mu \mathrm{L}$ dimethyl sulfoxide. Sodium borohydride $(3.3 \mu \mathrm{L} ; 50$ or $500 \mathrm{mM}$ ) was then added followed by $10 \mu \mathrm{L} 5^{\prime}$-IAF (10 $\mathrm{mg} \mathrm{mL}^{-1}$ ). HPLC injection volume was $20 \mu \mathrm{L}$ (equivalent to $2 \mu \mathrm{g}$ gliotoxin). C RP-HPLC analysis (method 3 ) of culture supernatants ( $48 \mathrm{~h}$; adjusted to $\mathrm{pH} 7.5$ ) of A. fumigatus Af293 followed reduction and subsequent labelling with $5^{\prime}$-IAF. The presence of GT-(AF) $)_{2}\left(R_{\mathrm{T}}\right.$, $16.79 \mathrm{~min}$ ) was observed. D RPHPLC analysis, using method 3, of culture supernatant $(48 \mathrm{~h}$; adjusted to $\mathrm{pH}$ 7.5) following addition of 5'-IAF $(20 \mu \mathrm{L}$; $\left.3 \mathrm{mg} \mathrm{mL}^{-1} ; 120 \mathrm{nmol}\right)$, without prior reduction. No GT-(AF) ${ }_{2}$ was evident. Free gliotoxin was observed $\left(R_{\mathrm{T}}, 15.49 \mathrm{~min}\right)$. Absorbance detection at $254 \mathrm{~nm}$
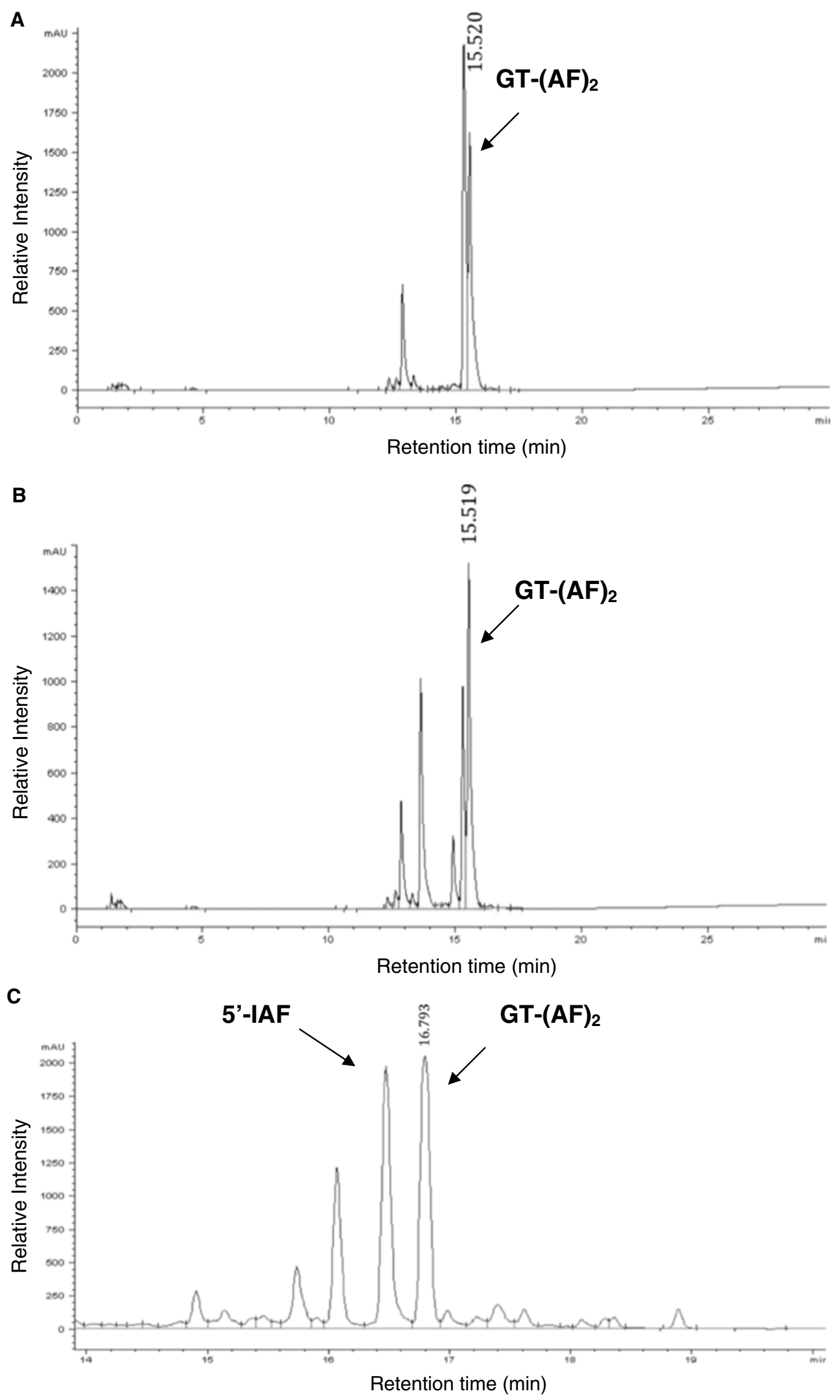


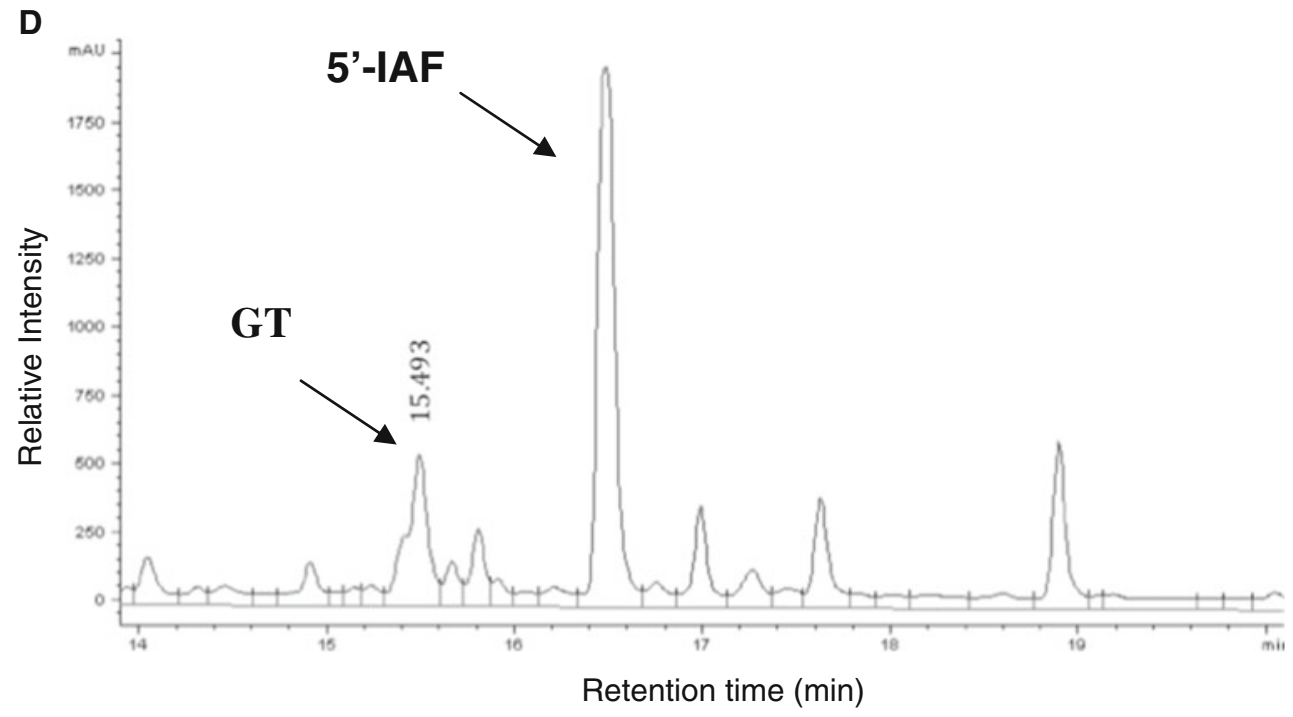

Fig. 6 (continued)

labelling has been preceded by $\mathrm{NaBH}_{4}$ reduction (Fig. S3, Electronic Supplementary Material).

\section{Discussion}

Improved methods for the sensitive and specific detection of gliotoxin have been developed. Firstly, it has been shown that the reducing agent, $\mathrm{NaBH}_{4}$, does not have to be removed prior to subsequent alkylation of gliotoxin, or sporidesmin A, thiols using 5'-iodoacetamidofluorescein (5'-IAF). Secondly, it appears that the resultant diacetamidofluorescein derivative of gliotoxin (GT-(AF) $)_{2}$ ) has a higher molar absorption (6.8fold) than unlabelled gliotoxin and so, should facilitate improved detection. Unlike free gliotoxin, the modified form can be detected by MALDI-ToF MS and has been shown to exhibit a molecular mass of 1103.93 Da. Our results also confirm that $A$. fumigatus-derived gliotoxin in organic extracts can be identified and that added- and nativegliotoxin is detectable in $\mathrm{pH}$-adjusted culture supernatants. In addition, it appears that $\mathrm{NaBH}_{4}$ is a superior gliotoxinreducing agent than DTT and that increased amounts of $\mathrm{NaBH}_{4}$ during modification steps may suppress unreacted 5'IAF interference during subsequent RP-HPLC analysis. Finally, we demonstrate that labelled gliotoxin can also be detected by TLC.

Conventional RP-HPLC methods, which use UV absorbance for detecting gliotoxin, depend solely on the $R_{\mathrm{T}}$ of gliotoxin in test specimens compared with that of a standard preparation $[10,20]$. While this approach is acceptable, the number of fungal metabolites which can be present in organic extracts, combined with the possibility of peptide generation due to unwanted proteolysis, demands a more rigorous confirmation of gliotoxin presence. LC-MS analysis of gliotoxin fulfils this criterion; however, this is a relatively specialised technique and is not available to all researchers [12, 15, 16]. Moreover, alternative bioassay formats have also been proposed for detection of gliotoxin and related epipolythiodioxopiperazines, of fungal origin, at levels of 18-20 ng well ${ }^{-1}[19,25]$. Although the sensitivity of the Microtox $^{\circledR}$ test is in the low micrograms-per-milliliter-range [25], the specificity of such tests, which deploy the sensitivity of bacterial luminescence to mycotoxin presence, as a measure of gliotoxin presence, is unclear. Sequential reduction and alkylation of gliotoxin, followed by detection using RP-HPLC, MALDI-ToF MS, or TLC represents a new direction for the improved detection of gliotoxin, whereby the molar absorption of the labelled form is almost sevenfold greater than free gliotoxin. This observation of enhanced sensitivity has not been forthcoming from elegant investigations of glutathione detection following modification with $N$-(1-pyrenyl)maleimide and subsequent HPLC identification of the resultant product [26]. Although GT-(AF) was fluorescent, it was observed that fluorescence quenching, under the acidic conditions employed for RP-HPLC identification, was apparent. Indeed, it has previously been noted that the quantum yield of fluorescein is significantly quenched in low $\mathrm{pH}$ environments [27] which further emphasises the practical significance of the enhanced absorbance of GT-(AF) 2 at 220-254 nm. 
$\mathrm{NaBH}_{4}$ reduction of protein disulphides prior to reaction with 4,4'-dithiodipyridine (Aldrithiol-4 ${ }^{\circledR}$ ) and subsequent thiol quantitation by detection of liberated 4-thiopyridone $\left(\mathrm{A}_{324 \mathrm{~nm}}\right)$ has been proposed to enable picomole detection of total protein thiols [28]. However, this approach required high temperature incubation $\left(50^{\circ} \mathrm{C}\right)$, addition of hexanol to prevent foaming during reduction, and destruction of residual $\mathrm{NaBH}_{4}$ by acidification, prior to 4, 4'-dithiodipyridine addition. The use of $\mathrm{NaBH}_{4}$ for gliotoxin reduction, which occurs instantaneously at room temperature $\left(20^{\circ} \mathrm{C}\right)$ requires none of these precautions. In fact, our results indicate that additional $\mathrm{NaBH}_{4}$ (tenfold) may actually assist the detection of the di-acetamidofluorescein derivative of gliotoxin by degrading free $5^{\prime}$-IAF present after reaction. In addition, the direct detection of the labelled gliotoxin product confers a degree of specificity not associated with 4-thiopyridone release which is an indirect measure of thiol presence and could, in theory, result from non-specific reduction of 4, 4'-dithiodipyridine.

The occurrence of ETP-encoding gene clusters and studies directed towards elucidation of gliotoxin biosynthesis, and toxicity, are the focus of significant current effort $[1,2,23,29-33]$. Thus, the development of a methodology for the specific detection of gliotoxin, especially under aqueous conditions and without the requirement for prior organic extraction and solvent removal, is significant because it enables direct identification of gliotoxin without specimen pre-treatment which may otherwise lead to gliotoxin loss or modification. Moreover, as with alternative strategies for mycotoxin detection [34], the reductive alkylation approach developed for detection of gliotoxin will also be useful in the detection of related ETP toxins such as sporidesmin $\mathrm{A}$, as we have demonstrated, and other disulphide- or thiol-containing molecules $[35,36]$.

Acknowledgements This work was funded by a Science Foundation Ireland Research Frontiers Project Grant (RFP/GEN/F571) and Enterprise Ireland (CFTD/2008/0141). SC was funded under Higher Education Authority (HEA) PRTLI-4. HPLC facilities were funded by the HEA, and MALDI-ToF MS was funded by the Health Research Board. We appreciate the input of Dr. John Stephens.

\section{References}

1. Kwon-Chung KJ, Sugui JA (2008) What do we know about the role of gliotoxin in the pathobiology of Aspergillus fumigatus? Med Mycol 47:S97-S103

2. Spikes S, Xu R, Nguyen CK, Chamilos G, Kontoyiannis DP, Jacobson RH, Ejzykowicz DE, Chiang LY, Filler SG, May GS (2008) Gliotoxin production in Aspergillus fumigatus contributes to host-specific differences in virulence. J Infect Dis 197:479-486
3. Gardiner DM, Waring P, Howlett BJ (2005) The epipolythiodioxopiperazine (ETP) class of fungal toxins: distribution, mode of action, functions and biosynthesis. Microbiology 151:1021-1032

4. Gardiner DM, Howlett BJ (2005) Bioinformatic and expression analysis of the putative gliotoxin biosynthetic gene cluster of Aspergillus fumigatus. FEMS Microbiol Lett 248:241-248

5. Waring P, Sjaarda A, Lin QH (1995) Gliotoxin inactivates alcohol dehydrogenase by either covalent modification or free radical damage mediated by redox cycling. Biochem Pharmacol 49:11951201

6. Tsunawaki S, Yoshida LS, Nishida S, Kobayashi T, Shimoyama T (2004) Fungal metabolite gliotoxin inhibits assembly of the human respiratory burst NADPH oxidase. Infect Immun 72:3373-3382

7. Yoshida LS, Abe S, Tsunawaki S (2000) Fungal gliotoxin targets the onset of superoxide-generating NADPH oxidase of human neutrophils. Biochem Biophys Res Commun 268:716-723

8. Jegorov A, Hajduch M, Sulc M, Havlicek V (2006) Nonribosomal cyclic peptides: specific markers of fungal infections. J Mass Spectrom 41:563-576

9. Mennink-Kersten MA, Klont RR, Warris A, Op den Camp HJ, Verweij PE (2004) Bifidobacterium lipoteichoic acid and false ELISA reactivity in Aspergillus antigen detection. Lancet 363:325-327

10. Reeves EP, Messina CG, Doyle S, Kavanagh K (2004) Correlation between gliotoxin production and virulence of Aspergillus fumigatus in Galleria mellonella. Mycopathologia 158:73-79

11. Watanabe A, Kamei K, Sekine T, Waku M, Nishimura K, Miyaji M, Tatsumi K, Kuriyama T (2004) Effect of aeration on gliotoxin production by Aspergillus fumigatus in its culture filtrate. Mycopathologia 157:245-254

12. Nieminen SM, Kärki R, Auriola S, Toivola M, Laatsch H, Laatikainen R, Hyvärinen A, Von Wright A (2002) Isolation and identification of Aspergillus fumigatus mycotoxins on growth medium and some building materials. Appl Environ Microbiol 68:4871-4875

13. Grovel O, Pouchus YF, Robiou du Pont T, Montagu M, Amzil Z, Verbist J (2002) Ion trap MS(n) for identification of gliotoxin as the cytotoxic factor of a marine strain of Aspergillus fumigatus Fresenius. J Microbiol Methods 48:171-179

14. Fox M, Gray G, Kavanagh K, Lewis C, Doyle S (2004) Detection of Aspergillus fumigatus mycotoxins: immunogen synthesis and immunoassay development. J Microbiol Methods 56:221-230

15. Lewis RE, Wiederhold NP, Chi J, Han XY, Komanduri KV, Kontoyiannis DP, Prince RA (2005) Detection of gliotoxin in experimental and human aspergillosis. Infect Immun 73:635637

16. Kupfahl C, Heinekamp T, Geginat G, Ruppert T, Härtl A, Hof H, Brakhage AA (2006) Deletion of the gliP gene of Aspergillus fumigatus results in loss of gliotoxin production but has no effect on virulence of the fungus in a low-dose mouse infection model. Mol Microbiol 62:292-302

17. Davis C, Carberry S, Schrettl M, Singh I, Stephens JC, Barry SM, Kavanagh K, Challis GL, Brougham D, Doyle S (2011) The role of glutathione $s$-transferase GliG in gliotoxin biosynthesis in Aspergillus fumigatus. Chem Biol 18:542-552

18. Peterson DS (2007) Matrix-free methods for laser desorption/ ionization mass spectrometry. Mass Spectrom Rev 26:19-34

19. Grovel O, Kerzaon I, Petit K, Robiou Du Pont T, Pouchus YF (2006) A new and rapid bioassay for the detection of gliotoxin and related epipolythiodioxopiperazines produced by fungi. J Microbiol Methods 66:286-293 
20. Bernardo PH, Chai CL, Deeble GJ, Liu XM, Waring P (2001) Evidence for gliotoxin-glutathione conjugate adducts. Bioorg Med Chem Lett 11:483-485

21. Bernardo PH, Brasch N, Chai CL, Waring P (2003) A novel redox mechanism for the glutathione-dependent reversible uptake of a fungal toxin in cells. J Biol Chem 278:46549-46555

22. Woodcock JC, Henderson W, Miles CO (2001) Metal complexes of the mycotoxins sporidesmin A and gliotoxin, investigated by electrospray ionisation mass spectrometry. J Inorg Biochem $85: 187-199$

23. Bok JW, Chung D, Balajee SA, Marr KA, Andes D, Nielsen KF, Frisvad JC, Kirby KA, Keller NP (2006) GliZ, a transcriptional regulator of gliotoxin biosynthesis, contributes to Aspergillus fumigatus virulence. Infect Immun 4:6761-6768

24. Carberry S, Neville CM, Kavanagh KA, Doyle S (2006) Analysis of major intracellular proteins of Aspergillus fumigatus by MALDI mass spectrometry: identification and characterisation of an elongation factor 1B protein with glutathione transferase activity. Biochem Biophys Res Commun 341:1096-1104

25. Alba P, Sánchez-Fortún S, Alvarez-Perez S, Blanco JL, García ME (2009) Use of a microbial toxicity test (Microtox) to determine the toxigenicity of Aspergillus fumigatus strains isolated from different sources. Toxicon 53:729-733

26. Hansen RE, Roth D, Winther JR (2009) Quantifying the global cellular thiol-disulfide status. Proc Natl Acad Sci USA 106:422-427

27. Hermanson GT (1996) Bioconjugate techniques. Academic, San Diego, p 302

28. Hansen RE, Østergaard H, Nørgaard P, Winther JR (2007) Quantification of protein thiols and dithiols in the picomolar range using sodium borohydride and 4,4'-dithiodipyridine. Anal Biochem 363:77-82

29. Patron NJ, Waller RF, Cozijnsen AJ, Straney DC, Gardiner DM, Nierman WC, Howlett BJ (2007) Origin and distribution of epipolythiodioxopiperazine (ETP) gene clusters in filamentous ascomycetes. BMC Evol Biol 7:174

30. Fox EM, Howlett BJ (2008) Biosynthetic gene clusters for epipolythiodioxopiperazines in filamentous fungi. Mycol Res 112:162-169

31. Choi HS, Shim JS, Kim JA, Kang SW, Kwon HJ (2007) Discovery of gliotoxin as a new small molecule targeting thioredoxin redox system. Biochem Biophys Res Commun 359:523-528

32. Balibar CJ, Walsh CT (2006) GliP, a multimodular nonribosomal peptide synthetase in Aspergillus fumigatus, makes the diketopiperazine scaffold of gliotoxin. Biochemistry 45:15029-15038

33. Kupfahl C, Michalka A, Lass-Flörl C, Fischer G, Haase G, Ruppert T, Geginat G, Hof H (2008) Gliotoxin production by clinical and environmental Aspergillus fumigatus strains. Int $\mathrm{J}$ Med Microbiol 298:319-327

34. Maragos CM (2009) Recent advances in the development of novel materials for mycotoxin analysis. Anal Bioanal Chem 395:12051213

35. Srinivasan U, Bala A, Jao SC, Starke DW, Jordan TW, Mieyal JJ (2006) Selective inactivation of glutaredoxin by sporidesmin and other epidithiopiperazinediones. Biochemistry 45:8978-8987

36. Rahman I, Kode A, Biswas SK (2006) Assay for quantitative determination of glutathione and glutathione disulfide levels using enzymatic recycling method. Nat Protoc 1:3159-3165 